\title{
Variation of Technological Adaptability Evaluated by the Performance and Brain Hemodynamics Measurement
}

\author{
Hajime Harada $^{1)}$, Kiyotaka Morozumi ${ }^{1)}$, Hiroshi Nashihara ${ }^{1)}$ and Eiko Hatakeyama ${ }^{2)}$ \\ 1) Department of Industrial Design, Tohoku Institute of Technology \\ 2) Kansei Fukushi Resarch Center, Tohoku Fukushi University
}

\begin{abstract}
This paper investigates the applicability of cerebral blood flow in evaluating the technological adaptability for operating industrial products. The procedure of the experiment was explained to the subjects and informed consent was obtained from them. Twenty male and twenty female subjects (19-22yrs) operated the destination setting task of a car navigation system. Subjects were divided into two sub groups to operate tasks of model A and model B of a car navigation system. Operation time of tasks and cerebral blood flow of frontal region were measured during tasks. Non-invasive measuring of regional cerebral blood flow was estimated by measuring deoxygenated hemoglobin, oxygenated hemoglobin, and total haemoglobin using the time resolved spectroscopy (TRS). Females were faster than males in operating the task of setting the destination searched by street address. Total haemoglobin of male subjects was significantly higher than that of females during resting and tasks. Changes of cerebral blood flow were observed during operating a car navigation system. In this paper we discussed the possibility of physiological evaluation for technological adaptability by means of the performance and brain hemodynamics measurement. J Physiol Anthropol Appl Human Sci 24(4): 383-388, 2005 http://www.jstage.jst.go.jp/browse/jpa
\end{abstract}

[DOI: 10.2114/jpa.24.383]

Keywords: hemodynamics measurement, techno-adaptability, industrial products

\section{Introduction}

Effects on humans with inhalation of odors (Miyazaki et al., 1999; Iijima et al., 2001; Tsunetsugu et al., 2002) and olfactory sensation of whisky and shochu (Morikawa et al., 2001; Morikawa et al., 2002) have been investigated by means of measuring regional cerebral blood flow using the nearinfrared spectroscopy (NIRS). Furthermore, the relationships between cerebral blood flow and subjective sensations to thermal stimuli (Iwanaga et al., 1999), the relationship between brain hemodynamics and EEG (Iwanaga et al., 2000), the effect of auditory stimuli on cerebral blood volume (Suzuki et al., 2000; Yamaguchi et al., 2001), the effect of oral stimuli of drinking coffee on cerebral blood volume (Hatakeyama et al., 2003) were investigated. We have conducted experiments on changes in brain hemodynamics for evaluation of the adaptability for driving (including stress and comfort) using a NIRS (Harada et al., 2002). The NIRS has the potential for imaging the sequence of brain activation (Hoshi and Tamura, 1997).

Recently, Tsunetsugu et al. (2004) reported that the time resolved spectroscopy (TRS) might be available for the evaluation of physiological polymorphism of humans. We have also applied the TRS to evaluate the adaptability for operating industrial products.

We are surrounded by many industrial products such as video or DVD player, microwave, washing machine, automobile and many other products with equipped microcomputer which we operate. However, we cannot ignore those products to operate in the urban life.

Furthermore, even when public transportations are available in our daily life, we need a car. However, we are always face with the possibility of traffic accident every day. Traffic accidents are caused by talking with passengers, controlling radio or $\mathrm{CD}$ player, looking at a car navigation monitor, talking on the handy phone, and many other reasons. We focused on the evaluation of performance and brain activity during operation of industrial products such as a car navigation system. Car navigation system is commonly used in Japan compared to other countries mainly because of our narrow and complicated roads, and frequent traffic jam in the city.

This paper investigates the applicability of the performance and brain hemodynamics in evaluating variation of the technological adaptability for operating industrial products such as a car navigation system by means of measuring operation time and brain activity using the time resolved spectroscopy. 


\section{Method}

\section{Subjects}

Twenty five male $(21.1 \pm 0.9)$ and twenty five female $(20.8 \pm 1.0)$ students volunteered as subjects in the present experiment. The procedure of the experiment was explained to the subjects and informed consent was obtained from them. Ten male $(21.1 \pm 0.9)$ and ten female subjects $(20.3 \pm 1.3)$ operated the task of a car navigation system of model A. The other fifteen male $(21.0 \pm 0.9)$ and fifteen female subjects $(21.0 \pm 0.6)$ operate the task of a car navigation system of model B.

\section{Tasks}

Tasks were designed using a software (Director 8.0J, Macromedia) and tasks were installed in a personal computer (FMV-BIBLO-NB75GA/T, Fujitsu, Japan) connected with a touch panel monitor (17inch LCD monitor, Touch Panel Systems, Japan). The tasks of model A were similarly designed as the product of car navigation system in the market. The tasks of model B were redesigned from the model A.

Figure 1 shows the top screen of a car navigation system of model A and model B. Task for model A consists of the following 6 tasks. Task 1 is to activate the system. Task 2 is to set the destination searched by street address, then to cancel the destination. Task 3 is set the destination searched by phone number, then to cancel the destination. Task 4 is to set the destination searched by genre, then to cancel the destination. Task 5 is to set the destination searched by name, then to cancel the destination. Task 6 is to turn the power off the system.

Details of Task 2 are to select 'navigation' from the menu (Fig. 1), to select 'destination' from the menu (Fig. 2), to select 'street address' from the menu (Fig. 3), and to input the street address of destination (Fig. 4). Model A consists of 7 menus (Fig. 2) and model B consists of three menus in Task 2 (Fig. 5). Task 3 is similar to task 2 except searching by phone number.

The subjects sit on a chair facing the touch panel monitor in the ordinary room. Figure 6 shows the picture of operating a simulated car navigation system in the present experiment.

Experiment was conducted under the room temperature of $25-28$ degrees Centigrade and relative humidity of $40-65 \%$.

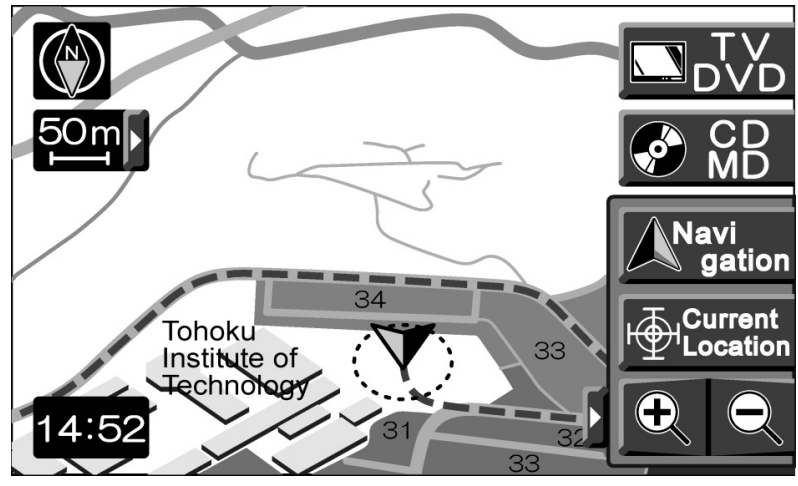

Fig. 1 The top screen of a car navigation system of model A.

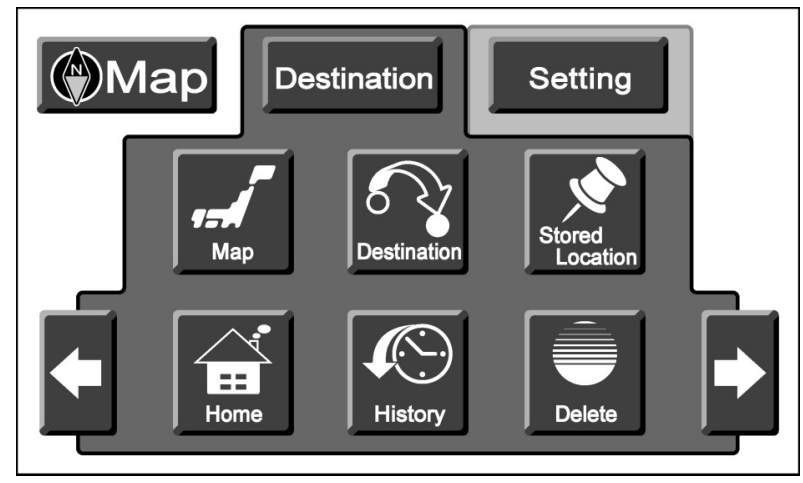

Fig. 2 Selection of 'destination' from the menu in task 2 of model A.

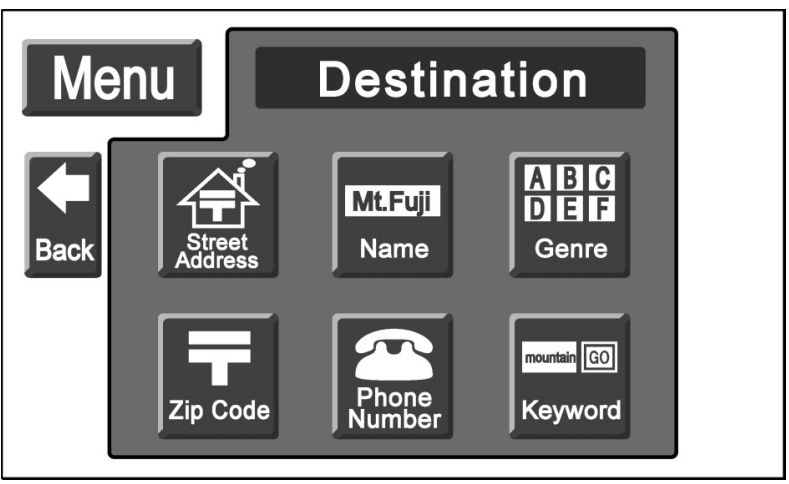

Fig. 3 Selection of 'street address' from the menu in task 2 of model A.

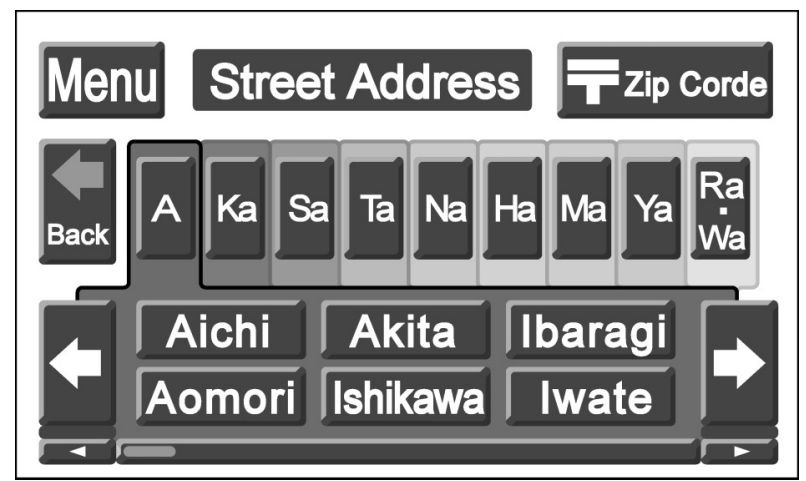

Fig. 4 Input of the street address in task 2 of model A.

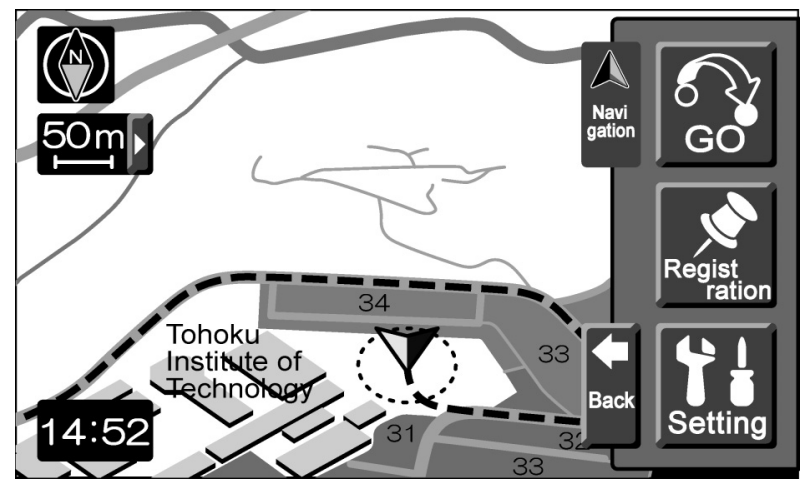

Fig. 5 A screen for navigation consisting of three menus in task 2 of model B. 


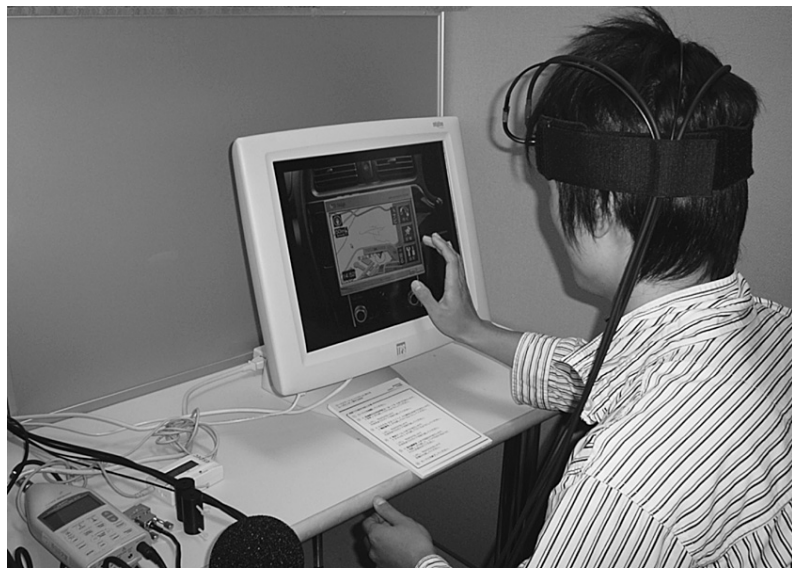

Fig. 6 Picture of operating a simulated car navigation system.

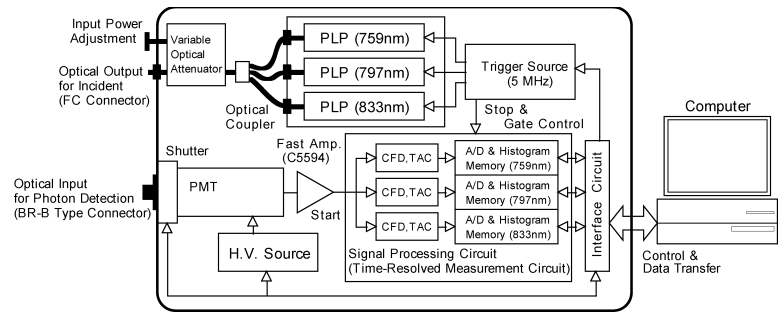

Fig. 7 Block diagram of time-resolved spectroscopy system (TRS-10). CFD: Constant Fraction Discriminator, MCP: Micro-channel Plate PHA: Pulse Height Analyzer PLP: Pico second Light Pulser PMT: Photomultiplier Tube, TAC: Time-to Amplitude Converter

Background noise was 45-50 dB (A) (40-47 dB) at resting state and $50-60 \mathrm{~dB}(\mathrm{~A})$ during task operations.

\section{Measurements}

We have measured the brain hemodynamics (cerebral blood flow of frontal region) using a near-infrared oxygenation monitor TRS-10 (Time Resolved Spectroscopy, Hamamatsu Photonics K.K., Japan) during the operation tasks of a car navigation system.

Figure 7 shows a block diagram of TRS-10 for tissue oxygen monitoring (Oda et al., 1999; Oda et al., 2000). The TRS-10 system consists of a three wavelength (760, 800, 830 $\mathrm{nm}$ ) of PLP (Pico second Light Pulser) as pulsed light source with a pulse width of around $100 \mathrm{ps}$, a high speed photomultiplier tube (PMT) with high sensitivity and three signal processing circuits for time-resolved measurement (CFD/TAC, A/D converter and histogram memory). Optical pulse train consisting of 760,800 and $830 \mathrm{~nm}$ is generated by $\mathrm{PLP}$ at $5 \mathrm{MHz}$ repetition rate and irradiated a sample through a single optical fiber. The diffuse-reflected light from the sample is collected by a bundle fiber and then detected by the PMT for single photon measurement. After being amplified by a following fast amplifier, a signal processing circuit integrated the TRS data for each wavelength individually.

Two fiber optic bundles (optodes), emitting and collecting near-infrared pulsed laser light, were fixed on both sides of the

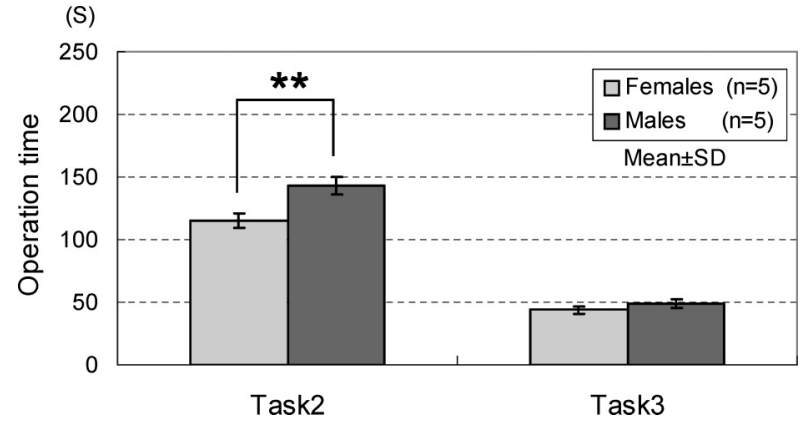

Fig. 8 Operation time of faster group in task 2 and task 3 of model A $\left({ }^{*} p<0.01\right)$.

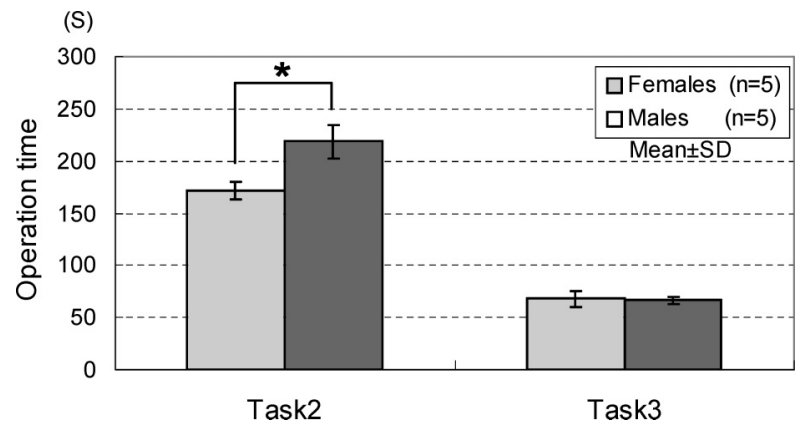

Fig. 9 Operation time of slower group in task 2 and task 3 of model A $\left({ }^{*} p<0.05\right)$.

subject's forehead with an interoptode distance of $45 \mathrm{~mm}$. Absolute concentration changes of oxygenated hemoglobin $\left(\mathrm{O}_{2} \mathrm{Hb}\right)$, deoxygenated hemoglobin $(\mathrm{HHb})$, and total hemoglobin $(\mathrm{tHb})$ were recorded using the TRS-10. Operation time of each task was calculated by a protocol analyses by means of video recording of task operation.

\section{Statistical analyses}

Non paired student's $t$-test was used to compare the operation time of model A and model B for both males and females. Hemodynamics changes were analyzed by two way ANOVA (models, tasks). Post-hoc comparisons of means were performed by means of a Fisher's protected least significant difference. Obtained values are shown as the mean and standard deviation (SD) for operation time and hemodynamics change. Statistical significance was accepted at the level of less than $p<0.05$.

\section{Results}

Subjects were divided into two groups according to their operation time in Task 2 of model A or model B. Figure 8 and 9 show the result of operation time in task 2 and task 3 of model A. Females of faster operation times showed significantly shorter time than that of males in task 2 of model A (Fig. 8, $p<0.01$ ). Females of slower operation times also showed significantly shorter time than that of males (Fig. 9, $p<0.05$ ). Operation time of females and males did not show 


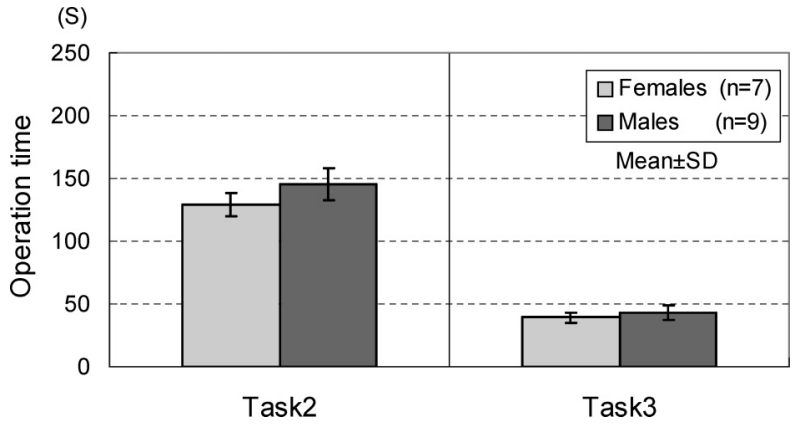

Fig. 10 Operation time of faster group in task 2 and task 3 of model B.

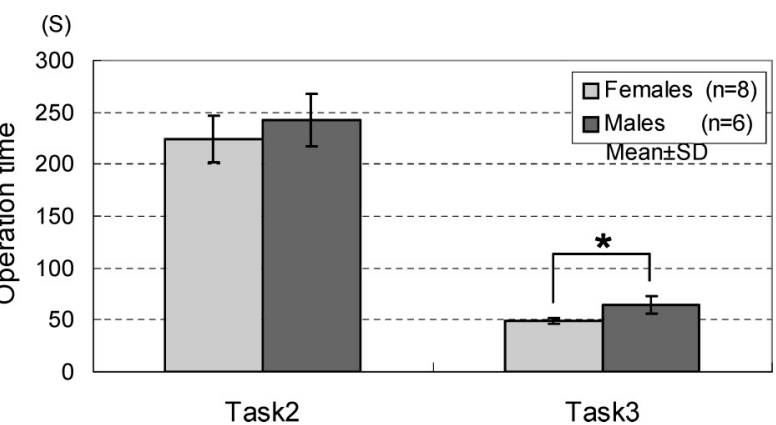

Fig. 11 Operation time of slower group in task 2 and task 3 of model B $\left({ }^{*} p<0.05\right)$.

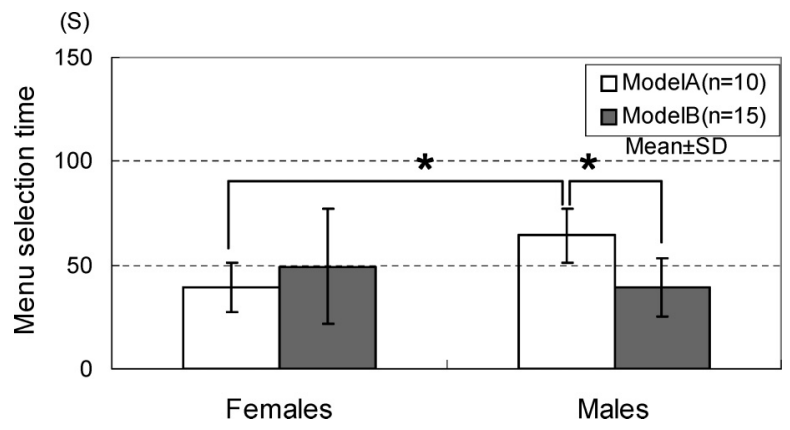

Fig. 12 Menu selection time out of operation time in task 2 of model A and model $\mathrm{B}(* p<0.05)$.

any significant difference in task 3 of model A.

As shown in Fig. 10, operation time in task 2 and task 3 of model B did not show any significant difference between females and males of faster operation time. The subjects of slower operation times did not show any significant difference between females and males in task 2 of model B (Fig. 11). Females of slower operation times showed significantly shorter time than that of males in task 3 of model B $(p<0.05)$.

Figure 12 shows the menu selection time out of operation time in task 2 of model A and model B. Females selected menu significantly faster than that of males in task 2 of model A $(p<0.05)$. However, there was no significant difference between females and males in task 2 of model B. Futhermore, males operated significantly faster to select menu in task 2 of model B than model A $(p<0.05)$.

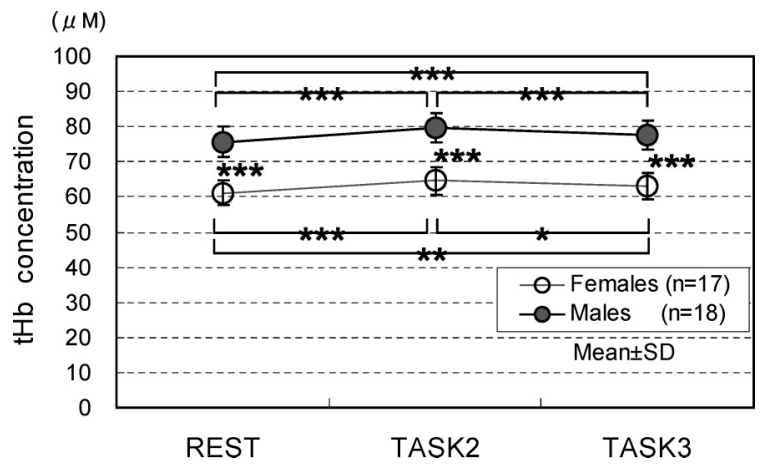

Fig. 13 tHb concentration changes of females and males at resting, task 2 , and task $3\left({ }^{*} p<0.05, * * p<0.01, * * * p<0.001\right)$.

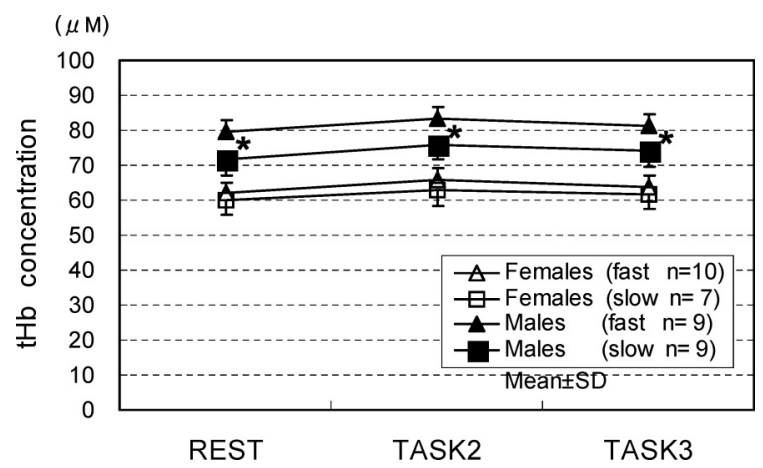

Fig. $14 \mathrm{tHb}$ concentration changes of females and males classified by means of operation time of task $2(* p<0.05)$.

$\mathrm{O}_{2} \mathrm{Hb}, \mathrm{HHb}$, and $\mathrm{tHb}$ of both sides of frontal region showed similar changes in the present study (not shown). In this report, we focused on the changes in $\mathrm{tHb}$ of left frontal region. The $\mathrm{tHb}$ concentration of seventeen females and eighteen males was analyzed. The main effect of tasks was significant on the tHb $(\mathrm{F}(1,2)=53.41, p<0.0001)$. As shown in Fig. 13, tHb concentration of left frontal region of females show significantly lower values than that of males at resting, during task 2 , and task $3(p<0.0001)$. The tHb concentration of females shows significantly higher values during operating task 2 compared to resting $(p<0.001)$ and task $3(p<0.05)$. The $\mathrm{tHb}$ concentration of males also shows significantly higher values during operating task 2 compared to resting $(p<0.001)$ and task $3(p<0.001)$. The main effect of models was not significant on the $\mathrm{tHb}$ concentration. The $\mathrm{tHb}$ concentration was compared between operation time of faster group and that of slower group for each of the females and males. Operation time of the faster group of males shows significantly higher values of the $\mathrm{tHb}$ concentration (Fig. 14, $p<0.05$ ).

\section{Discussion}

Females of faster operation times showed significantly shorter time than that of males in task 2 of model A (selecting right one from 7 items). Females of slower operation times also showed significantly shorter time than that of males in task 2 of 
model A. Females showed to be quick in making decision to select the right one from the menu of many items. Women usually obtain better scores on tests which require rapid matching or identification of designated stimuli. Operation time of females and males did not show any significant difference in task 3 which was similar to task 2. Menu selection time caused longer operation time for males in task 2 of model A. Male subjects seemed to consider the whole structures before their reactions. Therefore, they take time to select the right one. However, when the males once learn the process, they could operate faster. It is suggested that less item of menu could be easier for males to choose the right one at the beginning of menu selection.

Female subjects show significantly lower value of $\mathrm{tHb}$ than that of male subjects. This coincides with our previous study (Harada et al., 2004). The tHb of most of the subjects increased during operating task 2. However, some of them showed no changes. Tamura (1994) reported that cerebral blood flow did not change for a subject who was good at mathematics when he tackled a mathematical question. However, other subjects experienced an increase in cerebral blood flow under the same situation. In the present study, when the subjects once learn the process of the task (task 2), task difficulty would decrease with the similar task (task 3 ). The tHb concentration shows lower values in task 2 than task 3 . Therefore, measurement of cerebral blood flow could apply for the evaluation of the task difficulty or adaptability for each person. Furthermore, it was reported that multi-channel nearinfrared oxygenation monitoring could reveal the inter-area relationships in brain hemodynamics during short term memory task (Iwanaga, 2002). Accordingly, hemodynamics changes has a possibility to evaluate technological adaptability of operating industrial products.

Female subjects showed significantly lower values of blood flow of frontal region than that of male at resting. In the present study, the subjects were divided into sub groups to compare their physiological polymorphism of technological adaptability according to their operation time. Operation time of the faster group of males shows significantly higher values of the $\mathrm{tHb}$ concentration. Iwanaga (2004) reported that the study of techno-adaptability should treat physiological responses under the techno-stress from the point of view of whole-body coordination and functional potentiality and data should be classified into several types statistically as physiological polymorphism of techno-adaptability. Furthermore, Tsunetsugu et al. (2004) reported that personality could explain the individual differences in the physiological response. Therefore, it could be possible to apply performance and brain hemodynamics together with other parameters in evaluating the variation of technological adaptability for operating industrial products.

\section{Conclusion}

Hemodynamics changes of frontal region shows the significant differences between tasks of operation of a car navigation system. Female subjects showed significantly lower values of blood flow of frontal region than that of male at resting. Operation time of faster group of males shows significantly higher values of the $\mathrm{tHb}$ concentration.

Although we have to collect more data, it could be possible to apply performance and brain hemodynamics changes together with other parameters in evaluating the technological adaptability for operating industrial products.

Acknowledgments The authors express their gratitude to Mr. Ogaki T, and Ms. Oba R for their technical assistance and to the members of the department for their cooperation in the experiment. This research was supported in part by a Grant-inAid for Scientific Research (A) (2) No.15207025 from the Japan Society for the Promotion of Science.

\section{References}

Harada H, Morozumi K, Nashihara H, Hatakeyama E (2004) Physiological evaluation of techno-adaptability. J Physiol Anthropol Appl Human Sci 23: 358-359

Harada H, Sato T, Suzuki Y, Morozumi K, Nashihara H (2002) Physiological evaluation of adaptability for driving. J Physiol Anthropol Appl Human Sci 21: 321

Hatakeyama E, Ishikawa N, Hirabuki S, Kumamoto T, Kawai R, Miyazaki Y (2003) Brain oxy-hemoglobin changes while drinking coffee. J Physiol Anthropol Appl Human Sci 22: 249

Hoshi Y, Tamura M (1997) Near-infrared optical detection of sequential brain activation in the prefrontal cortex during mental tasks. Neuroimage 5(4 Pt 1): 292-297

Iijima A, Tanizaki M, Nakahara Y (2001) Effects of inhalation of essential oils on prefrontal hemodynamics by NIRS and senseory assessment. J Physiol Anthropol Appl Human Sci 20: 150

Iwanaga K (2004) Physiological Anthropology with the focus on techo-adaptability. J Physiol Anthropol Appl Human Sci 23: 58

Iwanaga K, Harada H, Katsuura K, Morikawa T, Miyazaki Y (1999) Psychological responses to thermal stimuli on a palm(II)-Effects on brain hemodynamics. J Physiol Anthropol Appl Human Sci 18: 189

Iwanaga K, Harada H, Katsuura K, Morikawa T, Miyazaki Y (2000) Relationship between brain hemodynamics and EEG. J Physiol Anthropol Appl Human Sci 19: 117

Iwanaga K, Shimomura Y, Katsuura K, Morikawa T, Miyazaki Y (2002) Evaluation of mental work load using multichannel measurement of brain haemodynamics by NIRS. J Physiol Anthropol Appl Human Sci 21: 303-304

Miyazaki Y, Morikawa T, Yamamoto N (1999) Effect of wooden odoriferous substance on humans. J Physiol Anthropol Appl Human Sci 18: 189

Morikawa T, Fujii T, Fujita N, Miyazaki Y (2002) Effect of stimulation to gustatory and olfactory sensation of cedar 
barrel-stored whisky on central and autonomic nervous activities. J Physiol Anthropol Appl Human Sci 20: 303

Morikawa T, Miyazaki Y (2002) Effect of stimulation to gustatory and olfactory sensation of malt whisky and barley shochu on humans. J Physiol Anthropol Appl Human Sci 21: 136

Oda M, Yamashita Y, Nakano T, Suzuki A, Shimizu K, Hirano I, Shimomura F, Ohmae E, Suzuki T, Tsuchiya Y (1999) Nearinfrared Time-Resolved Spectroscopy System for Tissue Oxygenation Monitor. SPIE 3597: 611-617

Oda M, Yamashita Y, Nakano T, Suzuki A, Shimizu K, Hirano I, Shimomura F, Ohmae E, Suzuki T, Tsuchiya Y (2000) Nearinfrared Time-Resolved Spectroscopy System for Tissue Oxygenation Monitor. SPIE 4160: 204-210

Suzuki Y, Hatakeyama E, Matsuzaki T, Morikawa T, Sueyoshi S, Miyazaki Y (2000) Effect of auditory stimuli on cerebral blood volume, blood pressure and sensory evaluation on humans. J Physiol Anthropol Appl Human Sci 19: 118

Tamura M, Hoshi Y (1994) Noninvasive optical function imaging for human brain activity. Oyo Buturi 63: 232-239

Tsunetsugu Y, Morikawa T, Miyazaki Y, Handa T, Hatakeyama E (2002) Relationship between personality and physiological response to odorous stimuli. J Physiol Anthropol Appl Human Sci 21: 303
Tsunetsugu Y, Morikawa T, Miyazaki Y, Kamiwaki T (2004) Personality and physiological response (2)-Relationship between trait anxiety, type A behaviour pattern and response to gustatory stimulation-. J Physiol Anthropol Appl Human Sci 23: 174

Tsunetsugu Y, Morikawa T, Miyazaki Y, Shimada M, Kamiwaki T, Oda M, Yamashita Y, Sato M (2004) Measurement of absolute $\mathrm{Hb}$ concentration in a prefrontal region by near-infrared time resolve spectroscopy. J Physiol Anthropol Appl Human Sci 23: 174

Yamaguchi M, Hatakeyama E, Kikuchi Y, Morikawa T, Sueyoshi S, Miyazaki Y (2001) Effect of auditory stimuli on cerebral blood volume-using near infrared spectroscopy (NIRS). J Physiol Anthropol Appl Human Sci 20: 150

Received: January 31, 2005

Accepted: March 14, 2005

Correspondence to: Hajime Harada, Department of Industrial Design Tohoku Institute of Technology, 35-1 Yagiyama Kasumi-cho, Taihaku-ku, Sendai, Miyagi 982-8577, Japan

Phone: +81-22-229-1151 (ext. 467)

Fax: +81-22-229-1545

e-mail: h-harada@tohtech.ac.jp 